\title{
Explorando las relaciones entre el desplazamiento al trabajo y los cambios de residencia en España. Un análisis con datos individuales*
}

\author{
Javier Romaní \\ AQR-IREA. Universitat de Barcelona \\ romani@ub.edu \\ José Manuel Casado-Díaz \\ Adelaida Lillo-Bañuls \\ Instituto Interuniversitario de Economía Internacional y Departamento de Análisis \\ Económico Aplicado. Universidad de Alicante \\ jmcasado@ua.es; alillo@ua.es
}

\begin{abstract}
Resumen
En este artículo, se analizan los factores determinantes de los tipos de movilidad más significativos que afectan a los trabajadores: los desplazamientos cotidianos entre la residencia y el lugar de empleo, y los cambios de domicilio. También se explora la influencia que cada uno de estos fenómenos ejerce en el otro. Para ello, se estiman dos modelos alternativos mediante la técnica del logit multinomial. Los datos individuales utilizados se refieren al caso español y proceden del Censo de Población de 2011, con lo que se actualizan estudios previos realizados antes de la gran recesión. Una aportación significativa del artículo es el uso de los mercados locales de trabajo (MLT) españoles como unidad territorial de referencia. Cada uno de dichos mercados agrupa a municipios estrechamente ligados entre sí en términos de movilidad entre la residencia y el lugar de empleo, y están delimitados por fronteras que son cruzadas con poca frecuencia en el curso de dichos desplazamientos, lo que los convierte en unidades funcionales relevantes frente a la alternativa de utilizar referencias geográficas de naturaleza administrativa. El uso de los MLT permite distinguir, a partir de los microdatos censales recodificados por el INE, entre movimientos migratorios (variaciones de lugar de residencia entre MLT distintos) y simples movimientos residenciales dentro de un mismo MLT (que, por tanto, no hacen imprescindible un cambio de ubicación laboral en términos de accesibilidad). Los resultados corroboran que existe una
\end{abstract}

* Esta investigación ha sido financiada por el proyecto CSO2014-55780-C3-2-P (Programa Estatal de Fomento de la Investigación Científica y Técnica de Excelencia, Ministerio de Economía y Competitividad). Los datos utilizados han sido facilitados por el Instituto Nacional de Estadística (INE), aunque dicha institución no es responsable de la exactitud de los análisis llevados a cabo por los autores. 
relación significativa entre los dos tipos de movilidad considerados y que las mudanzas a una nueva vivienda tienden a alejar a los empleados de su lugar de trabajo, en vez de acortar dichos desplazamientos.

Palabras clave: desplazamiento al lugar de empleo; cambio de residencia; mercado local de trabajo

Abstract. Exploring the Ties Between Commuting and Residence Change in Spain: An Analysis with Individual Data

This article discusses the determinants of two of the most significant types of workers' mobility: travel-to-work commuting and changes in the place of residence. It explores, in particular, the extent to which these phenomena influence each other. Accordingly, two alternative models are estimated using the multinomial logit technique. The individual data used come from the Spanish Population Census of 2011, which allows updating previous works that did not take into account the effects of the Great Recession. A significant contribution of the paper is the use of labour market areas (LMAs) as territorial reference units. Each of these areas brings together closely linked municipalities in terms of commuting flows and are delimited by boundaries that are infrequently crossed in the course of such trips; two features which make them relevant for this analysis in contrast with the alternative of using administrative territorial units as the reference geography. The recodification of the census microdata by the INE according to this territorial reference makes it possible to differentiate between migrations (change of residence involving different LMAs) and simple residential moves within a given LMA (which therefore make a change of workplace unnecessary due to the specific accessibility conditions in the LMA). The results confirm that there is a significant link between both types of mobility (commuting and migration/ residential mobility) and, in particular, that changes of residence seem to move workers away from their places of work rather than shortening their commuting trips.

Keywords: commuting; residential change; local labour market

\section{Sumario}
1. Introducción
4. Resultados
2. Los datos
5. Conclusiones
3. El modelo
Referencias bibliográficas

\section{Introducción}

La elección de los lugares de residencia y de ocupación laboral son decisiones que tienen una gran importancia en el bienestar de empleados y de familias e influyen en gran medida en la forma y en la transformación de las estructuras urbanas y sociales. Conjuntamente, ambas decisiones determinan también la duración y la distancia del desplazamiento diario al trabajo (lo que en inglés se conoce con el término de commuting), y ejercen una gran influencia en el resto de desplazamientos de los empleados y sus familias (los relacionados con las compras, el ocio o la educación, fundamentalmente). 
El objetivo principal del estudio que presentamos es analizar los factores que explican la movilidad desde dos perspectivas fundamentales: por una parte, la asociada con los desplazamientos por razones laborales (commuting) y, por otra, los cambios de lugar de residencia. Asimismo, teniendo en cuenta que ambas están relacionadas y que contribuyen a explicarse mutuamente, en esta investigación, se exploran además dichas interconexiones y sus efectos. Para ello, se utilizan datos individuales procedentes del Censo de Población de 2011. En el análisis, se presta, además, especial atención a la elección de la unidad territorial de referencia apropiada para el estudio. Es por ello que los datos originales han sido recodificados por el Instituto Nacional de Estadística, para acomodarlos a la realidad funcional de los mercados de trabajo en ámbitos locales, que la literatura identifica con los denominados mercados locales de trabajo (MLT). Se trata de la primera ocasión en que los MLT derivados del Censo de 2011 son utilizados en este tipo de investigación.

Las decisiones individuales que configuran el comportamiento de los ocupados en cuanto a su movilidad cotidiana relacionada con el empleo han sido objeto de análisis por un buen número de artículos en el ámbito de las ciencias sociales. Cabe destacar que los primeros modelos significativos en el ámbito de la economía y la geografía, como el modelo urbano tradicional (Alonso, 1964), obviaban buena parte de dicho problema, puesto que localizaban todos los puestos de trabajo en el centro de la ciudad, de manera que la única decisión relevante era la localización residencial. Marmolejo y Torres (2015) destacan que este marco teórico ha sido el más utilizado en este tipo de exploraciones y que sigue siendo válido actualmente, aunque destacan que la movilidad por causas no laborales (compras u ocio, entre otras) cobra cada vez más importancia.

Es sabido que los cambios de residencia suelen responder a diversas circunstancias domésticas, a modificaciones en el ciclo vital o a deseos de mejorar la situación familiar (véase, por ejemplo, Wagner y Mulder, 2015). Por su parte, los cambios de trabajo se realizan, generalmente, para aprovechar alguna oportunidad laboral. En lo que respecta a las decisiones migratorias, la corriente más clásica considera las diferencias salariales entre territorios como el principal factor explicativo de la migración (Greenwood, 1969; Greenwood y Hunt, 1989, 2003), mientras que otros autores apuntan a los desajustes entre el capital humano de los trabajadores y las cualidades requeridas por el mercado laboral en cada zona (Molho, 1986; Borjas et al., 1992; Faggian y McCann, 2009). Una corriente distinta subraya la relevancia del deseo de mejorar la calidad de vida como determinante de la decisión (Knapp y Graves, 1989; Mueser y Graves, 1995). Son relevantes, en este sentido, los trabajos de Del Pino (2014, 2015), quien subraya el carácter multidimensional del concepto de vivienda. Zax (1991 y 1994) y Zax y Kain (1991) destacan que, en cualquier caso, las situaciones personales y familiares pueden modificarse y convertir en subóptima una combinación entre residencia y lugar de empleo que previamente era satisfactoria. Para mejorar el bienestar en esas circunstancias, los trabajadores pueden optar por un cambio laboral (quit), por un cambio 
de residencia (move) o bien por una migración identificada como el traslado a un mercado de trabajo distinto, lo que implica una variación simultánea de residencia y de lugar de empleo (migration).

La literatura que relaciona, desde el punto de vista de las decisiones individuales, cambios de residencia y movilidad es abundante y no se ha alcanzado un consenso en cuanto a la direccionalidad ni a la significatividad de las distintas relaciones y variables analizadas. Así, por ejemplo, Shuai (2012) señala que, en el caso de las migraciones, no existe posibilidad de elegir la residencia y el puesto de trabajo de manera independiente (ambos se escogen de manera simultánea) ${ }^{1}$, mientras que las mudanzas de domicilio no implican necesariamente un reemplazamiento en la ubicación del empleo. En su estudio para el estado norteamericano de Virginia, este autor identifica un patrón según el cual las variaciones de residencia aumentan la proximidad al lugar de trabajo, con lo que disminuye el tiempo de desplazamiento. Este resultado contrasta con el de otros análisis también recientes, como los realizados por Axisa et al. (2012) y Newbold y Scott (2013), que se basan en el área metropolitana de Toronto; el de Mendiola et al. (2014), que estudia la provincia de Vizcaya, o el de Brown et al. (2015) que se ocupa del caso inglés. En este grupo de artículos, se concluye que los cambios de residencia aumentan el tiempo de desplazamiento al trabajo, lo que vendría explicado por la preferencia de las familias por residir en localizaciones suburbanas, periurbanas o incluso rurales. Cabe señalar, sin embargo, que esta podría no ser una situación estable, dado que, en muchas ocasiones, esta mudanza de hogar genera también un cambio de trabajo a medio plazo que disminuye la duración del desplazamiento entre el domicilio y el lugar de empleo. En este sentido, Vale (2013) plantea que podría existir un «intervalo de indiferencia» en el tiempo de desplazamiento, de tal forma que un cambio de residencia o de localización del puesto de trabajo solo provocarán una modificación posterior en la otra variable (o, en ocasiones, en el modo de transporte) si el nuevo tiempo de desplazamiento se encuentra por encima del extremo superior de dicho intervalo de indiferencia.

Las relaciones que se establecen entre las dos variables de interés del presente estudio - la movilidad cotidiana entre la vivienda y el trabajo, y la movilidad residencial - están influidas por una gran variedad de características, tanto de los individuos como de sus hogares y de los territorios en los que residen. Así, y comenzando por el nivel educativo, Wrede (2013) desarrolla un modelo teórico en el que los empleados de menor cualificación maximizan su utilidad residiendo y trabajando en la periferia, mientras que los de cualificación media residen en la periferia y se desplazan a trabajar al centro de las ciudades. Por su parte, los empleados de mayor cualificación viven y trabajan cerca de los centros urbanos. Otras variables que influyen en la decisión de escoger la residencia y el puesto de trabajo son la disponibilidad de medios de transporte (Mendiola et al., 2014), la edad del individuo (Wagner y Mulder, 2015), las características de la familia (Mendiola et al., 2014; Wagner y Mulder, 2015),

1. Han et al. (2013) también detectan esta situación. 
la calidad de vida en el municipio de residencia (Shuai, 2012) o la presencia de más de un trabajador en el hogar (Vale, 2013; Wagner y Mulder, 2015).

La relación entre la movilidad cotidiana entre el domicilio y el lugar de empleo y los cambios de residencia en España ha sido analizada por Romaní et al. (2003), quien ha utilizado datos individuales procedentes del Censo de Población de 1991. Dicho artículo encuentra una relación positiva entre ambas variables, de manera que los ocupados que han cambiado de residencia recientemente tienden a tener desplazamientos al trabajo más largos que los que no lo hacen, lo que coincide con los resultados obtenidos en estudios realizados en otros países, como los ya reseñados Axisa et al. (2012), Newbold y Scott (2013) y Brown et al. (2015), y también con los realizados con posterioridad en el caso español, como el de Mendiola et al. (2014). Romaní et al. (2003) concluyen también que existe una relación positiva entre la movilidad y el nivel de cualificación de los empleados, y que, además, las circunstancias familiares resultan ser estadísticamente significativas, especialmente la existencia de varios trabajadores en el mismo hogar.

Cabe destacar que el caso español muestra, respecto a dicho ámbito, características que lo hacen especialmente peculiar en relación con las de nuestro entorno internacional. De entre ellas, cabe destacar dos aspectos especialmente significativos en la relación analizada: por un lado, la edad de emancipación es relativamente elevada, lo que provoca que sea más común que en otros países encontrarse con hogares donde conviven más de dos trabajadores; por otro lado, pese al cambio de tendencia que parece observarse en los últimos años, el índice de viviendas en propiedad es aún significativamente más alto que en otros estados occidentales, y ese fenómeno podría actuar dificultando los procesos de cambios de residencia. Todo ello en el marco de una caída de los precios de la vivienda que, según autores como Bloze y Skak (2016), podría suponer un freno adicional a los cambios de domicilio por parte de los propietarios, pues significaría vender la residencia actual a un precio bastante inferior al que la adquirieron, con las pérdidas consiguientes. La verificación de esta hipótesis es muy probable en el caso de España, si bien los datos disponibles para este artículo no permiten contrastarla.

Las peculiaridades del caso español y el impacto de la gran recesión, cuyo efecto sobre su mercado de trabajo ha sido mucho más intenso que en el resto de economías avanzadas, justifican la pertinencia del análisis propuesto en este artículo. En él, además de estudiar la relación recíproca existente entre la movilidad entre residencia y trabajo y los cambios de domicilio - un fenómeno relevante por el impacto que tiene en el territorio y en la configuración de la realidad laboral y social de los hogares-, así como de actualizar los análisis disponibles hasta ahora para el caso español, se realiza una aportación metodológica significativa. Dicha aportación está relacionada con la adopción, como unidad de análisis, de una geografía de unidades territoriales de carácter funcional, los mercados locales de trabajo (MLT), que permiten soslayar algunas de las limitaciones asociadas al uso de entidades de tipo administrativo, como los municipios o las comarcas, cuya idoneidad para el análisis de fenómenos 
sociales y económicos no ha sido contrastada, ya que son fruto de una lógica distinta.

En particular, en el presente estudio, se hace uso de una delimitación de varios MLT construidos como espacios dentro de los cuales es posible, en términos de accesibilidad, cambiar de lugar de trabajo sin modificar el lugar de residencia y, simultáneamente, es posible cambiar de lugar de residencia sin que ello obligue a modificar de lugar de trabajo. Desde un punto de vista operativo, tales mercados se construyen por la agregación de municipios intensamente vinculados entre sí en términos de movilidad entre residencia y lugar de trabajo, de tal forma que las fronteras exteriores de cada uno de estos MLT se cruzan diariamente con un volumen relativamente pequeño de ocupados en sus desplazamientos entre el domicilio y el trabajo. Es decir, se trata de regiones funcionales cohesionadas desde el punto de vista interno y relativamente autónomas desde el punto de vista externo. El uso de esta referencia territorial permite discriminar entre cambios de lugar de residencia que constituyen verdaderas migraciones («saltos» entre MLT distintos) y aquellos otros que son meros ajustes residenciales dentro de un mismo MLT (movimientos que, por tanto, hacen mucho menos necesarios los cambios de lugar de empleo desde el punto de vista de la accesibilidad).

El resto del artículo se estructura como sigue. En la siguiente sección, se describen los datos utilizados en el análisis, procedentes del Censo de Población de 2011, y la forma en que los microdatos censales han sido recodificados por el INE de acuerdo con la geografía propuesta. En el apartado 3, se especifican los dos modelos que serán estimados, con el fin de identificar qué factores resultan relevantes, y en qué sentido, a la hora de explicar las decisiones individuales relacionadas con la movilidad entre residencia y trabajo, por un lado, y con el cambio de domicilio, por otro. En ambos casos, se incluye como variable explicativa la otra forma de movilidad, junto con unas variables distintas sugeridas por la literatura. Ambos modelos se estiman mediante la técnica del logit multinomial. En el apartado siguiente, el número 4, se presentan y se discuten los resultados de las estimaciones. Por último, en el apartado de conclusiones, se sintetizan algunos de los principales resultados, se discuten las limitaciones del estudio y se proponen algunas posibles extensiones del mismo.

\section{Los datos}

Un problema para el estudio del commuting, los cambios de residencia y la migración en España es la inexistencia de una base de microdatos de tipo longitudinal, que refleje los distintos cambios de residencia y de lugar de empleo de un mismo trabajador a lo largo del tiempo, así como las modificaciones que hayan tenido lugar en sus características familiares ${ }^{2}$. Este tipo de datos sí que

2. Por ejemplo, Brown et al. (2015) trabajan con una base de datos de tipo longitudinal, lo cual les permite analizar si un cambio de residencia o de trabajo conduce a un cambio también de la otra variable (trabajo o residencia, respectivamente) a medio plazo. 
existen en Estados Unidos o en el Reino Unido. A pesar de esta deficiencia en la información, el análisis conjunto del commuting, los cambios de residencia y las migraciones puede realizarse, al menos parcialmente, a partir de los censos de población, tal como hacen Romaní et al. (2003). Sin embargo, se ven obligados a utilizar como criterio identificador de una migración el cambio del municipio de residencia, lo cual constituye una importante limitación, dado que, en muchos casos, este se produce entre municipios que pertenecen a un mismo mercado local de trabajo, de manera que es posible cambiar de residencia manteniendo el empleo. Una segunda limitación de los censos de población en España es que no recogen las variaciones de localización del lugar de empleo.

Por último, el tiempo transcurrido desde el Censo de Población de 1991 y los cambios económicos y sociales transcurridos en España desde dicho año justifican la realización de un análisis con datos más recientes, en concreto, los de la muestra de microdatos del Censo de Población y Viviendas de 2011. Se han seleccionado únicamente aquellas observaciones correspondientes a personas con un trabajo remunerado en el momento de realizar el censo. Por tanto, han sido eliminadas las que están inactivas o sin ocupación en dicho momento, dado que, al carecer de empleo, no pueden desplazarse hasta él.

$\mathrm{Tal}$ como se ha señalado anteriormente, los trabajos previos realizados en España sobre el commuting y los cambios de residencia con datos individuales (entre otros, Artís et al., 2000; Romaní et al., 2003; Casado-Díaz, 2000a y 2001; Romaní y Casado-Díaz, 2010) se encuentran con algunas limitaciones debidas al tipo de datos disponibles. Algunas de ellas son comunes a todos los trabajos que utilizan datos de corte transversal ${ }^{3}$, mientras que otras se deben a la necesidad de utilizar las fronteras de tipo administrativo - las de los municipios- para definir el carácter de los desplazamientos y los cambios de residencia. La realidad de la sociedad española contemporánea pone de manifiesto que tales límites administrativos se ven completamente desbordados por la naturaleza y la magnitud de fenómenos socioeconómicos de todo tipo, incluyendo los flujos de personas, especialmente en ámbitos fuertemente urbanizados. Así, en estudios realizados a partir de este tipo de movilidad, tanto Aguilera y Mignot (2004) como Marmolejo y Torres (2015) destacan la importancia de la estructura urbana (monocéntrica o policéntrica) sobre los flujos de movilidad en Francia y en España, respectivamente.

Para considerar el hecho de que la realidad funcional excede los límites administrativos, se solicitó al Instituto Nacional de Estadística una recodificación de los microdatos del Censo de Población y Viviendas de 2011, de acuerdo con la delimitación de los MLT en España realizada a partir de esa misma fuente estadística por Martínez-Bernabeu y Casado-Díaz (2016), utilizando el método propuesto en Martínez-Bernabeu et al. (2012) y Casado-Díaz et al. (2017). De esta manera, con los nuevos microdatos, es posible señalar si cada desplazamiento al trabajo se realiza dentro de un mismo MLT o entre

3. Una excepción es Arévalo et al. (2008), que trabajan con el Panel de Hogares de la Unión Europea (PHOGUE). 
dos MLT diferentes. Del mismo modo, podemos identificar si un cambio de residencia se ha producido dentro del mismo MLT (con lo cual podríamos identificarlo como un move, siguiendo la definición de Zax, 1991 y 1994, y Zax y Cain, 1991) o si se produce entre dos mercados locales diferentes, con lo cual podemos considerarlo una migración, según dichos autores.

De acuerdo con Goodman (1970), un MLT representa la realidad funcional del mercado de trabajo en el ámbito local y se define como una agrupación de unidades territoriales de partida (en el caso español, se trata de los municipios) que presentan las características siguientes:

a) Consideradas en su conjunto, se muestran relativamente aisladas frente al exterior en cuanto a los desplazamientos entre residencia y lugares de trabajo.

b) Registran un intercambio significativo de ocupados entre ellas de manera cotidiana.

Cada uno de los MLT en que puede dividirse un territorio se caracteriza, por tanto, porque es autónomo frente al exterior y cohesionado desde el punto de vista de las relaciones entre las unidades territoriales que lo constituyen. Existe una abundante literatura sobre el modo cómo el concepto de MLT puede aplicarse en la práctica, especialmente en el ámbito de las autoridades públicas y de los institutos nacionales de estadística, sobre todo europeos (véase Casado y Coombes, 2011, y Coombes et al., 2012). Entre ellos, destacan los métodos desarrollados en el Reino Unido por Coombes para definir el mapa oficial de las allí denominadas Travel-to-Work Areas o TTWAs (Coombes et al., 1986; Coombes y Bond, 2008), que en España fueron aplicados por Casado-Díaz (2000b) y Casado et al. (2010) y utilizados por Feria et al. (2015) para definir submercados de trabajo en el seno de las áreas metropolitanas. En los últimos años, se ha desarrollado, en el ámbito académico, una nueva familia de procedimientos de delimitación de MLT que ha mostrado su superioridad frente a las alternativas preexistentes (véanse las evaluaciones relativas realizadas en, por ejemplo, Casado-Díaz et al., 2017). Se trata de métodos que siguen los principios reconocidos internacionalmente, incluyendo los recomendados por Eurostat (Casado y Coombes, 2011; Coombes et al., 2012), pero que abordan la delimitación como un problema de optimización que es resuelto mediante técnicas de computación evolutiva ${ }^{4}$. A partir de estos procedimientos, la delimitación de MLT definida por Martínez-Bernabéu y Casado-Díaz (2016) divide España en 261 áreas funcionales adecuadas para el análisis del mercado de trabajo. Se trata de una geografía que recoge el territorio español de manera exhaustiva y excluyente: todos los municipios se asignan a un (y solo uno) MLT. El resultado es una delimitación de los MLT de tal forma que:

4. El uso de este tipo de técnicas permite también complementar la aplicación de métodos tradicionales, como el de las TTWA (véase Casado-Díaz et al., 2016). 
a) Se maximiza la cohesión interna de dichos mercados (garantizando que se trata de unos MLT cuyos municipios están intensamente relacionados entre sí en términos de movilidad entre los lugares de residencia y de trabajo).

b) Todos ellos superan un mínimo de autonomía del 70\% (es decir, para cada uno de los MLT, al menos el 70\% de los ocupados residentes trabajan dentro de sus límites y, simultáneamente, al menos el 70\% de los empleos localizados en el MLT son ocupados por personas que residen en el interior de dicho MLT).

Además, y con el fin de cumplir con los requisitos de confidencialidad y significatividad fijados por el INE, cada uno de los MLT tiene un tamaño poblacional mínimo de 20.000 habitantes.

La muestra de microdatos del Censo de Población de 2011 recodificados por el INE de acuerdo con este nuevo conjunto de referencias territoriales de trabajadores ocupados y sin datos incompletos constituye nuestra base de datos para la realización del análisis empírico que se desarrolla en el apartado 4. Se trata de un total de 1.483 .557 observaciones. Este es, por tanto, el tamaño muestral utilizado en nuestro análisis.

\section{El modelo}

Tal y como se ha señalado, para analizar el objetivo propuesto en el presente artículo, esto es, los factores que explican la movilidad, se estiman dos modelos. Uno desde la perspectiva de los desplazamientos por razones laborales y otro desde la de los cambios en el lugar de residencia.

En el primer modelo, la variable dependiente es el commuting y en el segundo, la movilidad residencial. En ambos modelos, la otra variable aparece como independiente junto con el resto de variables consideradas. Por desgracia, el Censo de Población de 2011 no proporciona información relativa a las modificaciones habidas en los lugares de empleo (ya sea un cambio de empresa o un cambio en la localización del puesto de trabajo), lo cual supone una limitación en nuestro estudio.

Así, en primer lugar, a partir de los datos relativos al desplazamiento al trabajo, commuting, que aparecen en el Censo de 2011, podemos construir las siguientes categorías:

1. Trabajadores que residen y trabajan en el mismo municipio.

2. Empleados que residen y trabajan en diferentes municipios del mismo mercado local de trabajo.

3. Empleados que trabajan y residen en distintos mercados locales de trabajo.

En segundo lugar, para los cambios de residencia, se pueden definir las siguientes categorías:

1. Trabajadores que no han cambiado de municipio de residencia entre 2001 y 2011. 
2. Empleados que cambiaron de municipio de residencia dentro de un mismo mercado local de trabajo (el planteamiento de la pregunta la limita a los cinco años anteriores al censo, es decir, solo detectaremos esta situación si el cambio de residencia se produjo entre 2007 y 2011).

3. Empleados que cambiaron su residencia de un mercado local de trabajo a otro diferente (el planteamiento de la pregunta provoca que solo podamos detectar estos cambios de residencia si se produjeron en el año inmediatamente anterior a la realización del censo).

Dada la construcción de las variables dependientes consideradas, la forma funcional más adecuada para ambas ecuaciones es un modelo logit multinomial $^{5}$, con tres categorías para la variable dependiente en cada ecuación. En la ecuación de commuting, la categoría de referencia (omitida) está formada por los trabajadores que no se desplazan fuera de su municipio de residencia, mientras que, en la ecuación de cambio de residencia, constarán los trabajadores que no han cambiado de domicilio.

Por último, las variables explicativas serán, en primer lugar, y tal y como se ha señalado, la variable dependiente del otro modelo, esto es, el cambio de residencia (en la ecuación de commuting) y el desplazamiento al trabajo (en la ecuación de cambio de residencia). Además, se añade un exhaustivo conjunto de variables de control relacionadas con las características personales, familiares, profesionales, de la vivienda y del tipo de transporte que utilizan los trabajadores. Se trata de variables que han sido señaladas como relevantes por los trabajos previos que se han ocupado de este tema (véase el apartado 1).

Así, en el primer modelo, commuting, las variables explicativas que, adicionalmente a la dependiente del otro modelo, $\operatorname{movilidad}^{6}$, han sido consideradas, incluyen tanto características personales, familiares y laborales como características de la vivienda. Entre las primeras, figuran variables como la edad, los años de estudios, el sexo, el estado civil, el cuidado de hijos menores de 15 años, la realización de trabajos en el hogar, el empleo a tiempo completo o parcial y la categoría profesional. Entre las características de la vivienda, se cuentan el número de habitaciones, el tamaño del hogar, si la vivienda es en propiedad o no, así como si cuenta con calefacción, saneamiento o el acceso a Internet ${ }^{7}$.

5. Se trata de una generalización del conocido modelo logit binario para el caso de que la variable dependiente (cualitativa) admita más de dos valores posibles. Uno de ellos se toma como categoría de referencia y se calculan las probabilidades del resto de valores contra la categoría de referencia. Reescalando luego dichas probabilidades de manera que la suma total sea 1 , se puede calcular la probabilidad de cada uno de los valores de la variable dependiente (ver Gujarati y Porter, 2010, o Greene, 2012, para una explicación más detallada de dicho modelo).

6. Variable categórica de cambio de residencia, siendo la categoría de referencia los que no han cambiado de residencia.

7. Se incluyen también en el modelo como variables de control las relativas al número de desplazamientos diarios, al modo de desplazamiento al trabajo y al sector de actividad, aunque estas y sus coeficientes no se muestran en las tablas con el fin de sintetizar la información presentada. Los resultados están disponibles. 
En lo que respecta al segundo modelo, cambio de residencia, las variables explicativas que han sido consideradas adicionalmente a la dependiente del otro modelo, commuting, incluyen de nuevo características personales, familiares y laborales, así como características de la vivienda. Entre las primeras, están la edad, el número de hijos, los años de estudios, el sexo, el estado civil, el cuidado o no de hijos menores de 15 años, la realización de tareas en el hogar y si el trabajo es a tiempo completo o parcial, así como la categoría profesional. Se amplían respecto al modelo anterior las variables relacionadas con las características de la vivienda, incluyendo información relativa a la superficie de la misma, la cantidad de habitaciones, de plazas de garaje, si existe o no ascensor, el número de personas ocupadas, si hay personas extranjeras en el hogar, si la vivienda es en propiedad o no, si cuenta con calefacción, saneamiento y acceso a Internet, así como el estado en que se encuentra el edificio (dado que consideramos que dichas variables tienen importancia en el momento de elegir la vivienda, pero no en el de localización del puesto de trabajo). También, como en el caso anterior, se incluyen en el modelo las variables de control relativas al número de desplazamientos diarios, el tipo de desplazamiento al trabajo y el sector de actividad.

Dado el elevado número de variables en los modelos, lo que, a priori, aumenta el riesgo de aparición de problemas de multicolinealidad, se han calculado los factores de inflación de la varianza (VIF, según sus siglas en inglés) en ambas ecuaciones. En ambos modelos, el VIF medio se encontraba ligeramente por debajo de 1,5 y en ningún caso se obtuvieron valores del VIF mayores que 3,2, muy inferiores al valor de 10 que se suele tomar como umbral para considerar la existencia de un problema de multicolinealidad importante (Gujarati y Porter, 2010).

\section{Resultados}

Los coeficientes obtenidos en las estimaciones de las ecuaciones 1 y 2 se recogen en las tablas 1 (ecuación de commuting) y 2 (ecuación de cambio de residencia) ${ }^{8}$.

Comenzando por el análisis de la variable de interés, la tabla 1 muestra que existe una relación significativa entre el commuting y los cambios de residencia: los trabajadores que han cambiado de residencia recientemente (ya sea a otro municipio del mismo mercado local de trabajo o a uno distinto) tienen unas probabilidades significativamente mayores de estar empleados fuera del municipio de residencia (ya sea en otro municipio del mismo mercado local de trabajo o en otro mercado local de trabajo) que quienes no han cambiado su municipio de residencia. Por su parte, quienes disponen de un domicilio en

8. Ambos modelos muestran un buen ajuste, con un pseudo $\mathrm{R}^{2}$ de McFadden cercano al 0,15 en ambos casos. Por su parte, el pseudo $\mathrm{R}^{2}$ en la ecuación de commuting es de 0,285 (Nagelkerke) y 0,147 (McFadden), y en la ecuación de cambio de residencia es de 0,184 (Nagelkerke) y 0,150 (McFadden). 


\begin{tabular}{|c|c|c|c|c|c|c|}
\hline & \multicolumn{3}{|c|}{$\begin{array}{l}\text { Entre municipios } \\
\text { del mismo MLT }\end{array}$} & \multicolumn{3}{|c|}{$\begin{array}{l}\text { Trabaja y reside } \\
\text { en diferentes MLT }\end{array}$} \\
\hline & Coef. & Signif. & Odds ratio & Coef. & Signif. & Odds ratio \\
\hline Interceptación & $-0,099$ & 0,008 & & $-0,728$ & 0,000 & \\
\hline Cambio de residencia entre varios MLT & 1,220 & 0,000 & 3,387 & 0,123 & 0,000 & 1,131 \\
\hline Cambio de residencia entre municipios del mismo MLT & 0,064 & 0,019 & 1,066 & 1,020 & 0,000 & 2,772 \\
\hline Edad & $-0,012$ & 0,000 & 0,988 & $-0,027$ & 0,000 & 0,974 \\
\hline Años de estudios & 0,012 & 0,000 & 1,012 & 0,032 & 0,000 & 1,033 \\
\hline Hombre & 0,241 & 0,000 & 1,273 & 0,385 & 0,000 & 1,470 \\
\hline Mujer & \multicolumn{6}{|c|}{ Categoría de referencia } \\
\hline Soltero & $-0,014$ & 0,011 & 0,986 & 0,061 & 0,000 & 1,062 \\
\hline Viudo & $-0,126$ & 0,000 & 0,882 & $-0,147$ & 0,000 & 0,863 \\
\hline Separado & 0,053 & 0,000 & 1,054 & 0,109 & 0,000 & 1,115 \\
\hline Divorciado & 0,030 & 0,003 & 1,030 & 0,035 & 0,028 & 1,035 \\
\hline Casado & \multicolumn{6}{|c|}{ Categoría de referencia } \\
\hline Cuidar a un menor de 15 años: sí & 0,024 & 0,000 & 1,025 & $-0,111$ & 0,000 & 0,895 \\
\hline Cuidar a un menor de 15 años: no & \multicolumn{6}{|c|}{ Categoría de referencia } \\
\hline Trabajos del hogar: sí & $-0,035$ & 0,000 & 0,966 & $-0,056$ & 0,000 & 0,945 \\
\hline Trabajos del hogar: no & \multicolumn{6}{|c|}{ Categoría de referencia } \\
\hline Trabajo a tiempo completo & 0,139 & 0,000 & 1,149 & 0,305 & 0,000 & 1,356 \\
\hline Trabajo a tiempo parcial & \multicolumn{6}{|c|}{ Categoría de referencia } \\
\hline Número de habitaciones de la vivienda & 0,004 & 0,005 & 1,004 & 0,038 & 0,000 & 1,039 \\
\hline Superficie útil & 0,002 & 0,000 & 1,002 & 0,002 & 0,000 & 1,002 \\
\hline Tamaño del hogar & $-0,001$ & 0,611 & 0,999 & $-0,017$ & 0,000 & 0,983 \\
\hline Vivienda: propia, adquirida por compra, totalmente pagada & $-0,160$ & 0,000 & 0,852 & $-0,149$ & 0,000 & 0,862 \\
\hline Vivienda: propia, adquirida por herencia o donación & $-0,126$ & 0,000 & 0,882 & 0,145 & 0,000 & 1,155 \\
\hline Vivienda: alquilada & $-0,326$ & 0,000 & 0,722 & $-0,546$ & 0,000 & 0,579 \\
\hline Vivienda: cedida gratis 0 a bajo precio & $-0,295$ & 0,000 & 0,744 & $-0,147$ & 0,000 & 0,863 \\
\hline Vivienda: otra forma de adquisición & $-0,078$ & 0,000 & 0,925 & 0,112 & 0,000 & 1,118 \\
\hline Vivienda: propia, por compra, con pagos pendientes (hipotecas) & \multicolumn{6}{|c|}{ Categoría de referencia } \\
\hline Calefacción central & $-0,139$ & 0,000 & 0,870 & $-0,391$ & 0,000 & 0,676 \\
\hline Calefacción individual & 0,228 & 0,000 & 1,257 & 0,102 & 0,000 & 1,107 \\
\hline Estufas & 0,029 & 0,000 & 1,029 & 0,057 & 0,000 & 1,058 \\
\hline Sin calefacción & \multicolumn{6}{|c|}{ Categoría de referencia } \\
\hline Baño o ducha: sí & 0,022 & 0,511 & 1,022 & $-0,052$ & 0,320 & 0,950 \\
\hline Baño o ducha: no & \multicolumn{6}{|c|}{ Categoría de referencia } \\
\hline Acceso a Internet: sí & $-0,010$ & 0,039 & 0,991 & $-0,418$ & 0,000 & 0,658 \\
\hline Acceso a Internet: no & \multicolumn{6}{|c|}{ Categoría de referencia } \\
\hline Agua caliente central: sí & 0,130 & 0,000 & 1,139 & 0,275 & 0,000 & 1,317 \\
\hline Agua caliente central: no & \multicolumn{6}{|c|}{ Categoría de referencia } \\
\hline Administrativos & 0,140 & 0,000 & 1,150 & $-0,005$ & 0,655 & 0,995 \\
\hline $\begin{array}{l}\text { Artesanos y trabajadores cualificados de las industrias manufactureras } \\
\text { y de la construcción }\end{array}$ & 0,058 & 0,000 & 1,060 & $-0,019$ & 0,148 & 0,981 \\
\hline Directivos & 0,103 & 0,000 & 1,109 & 0,178 & 0,000 & 1,195 \\
\hline Ocupaciones elementales & 0,086 & 0,000 & 1,090 & $-0,108$ & 0,000 & 0,898 \\
\hline Operadores de instalaciones y maquinaria, y montadores & 0,317 & 0,000 & 1,372 & 0,222 & 0,000 & 1,248 \\
\hline Técnicos y profesionales científicos e intelectuales & 0,090 & 0,000 & 1,094 & 0,236 & 0,000 & 1,267 \\
\hline Técnicos; profesionales de apoyo & 0,294 & 0,000 & 1,342 & 0,302 & 0,000 & 1,352 \\
\hline Trabajadores cualificados en el sector agrícola, ganadero, forestal y pesquero & $-0,300$ & 0,000 & 0,741 & $-0,485$ & 0,000 & 0,616 \\
\hline Servicios de restauración, personales, protección y vendedor & \multicolumn{6}{|c|}{ Categoría de referencia } \\
\hline
\end{tabular}

\section{Fuente: Censo de Población 2011, INE}

Nota: Nivel de significación: ${ }^{* \star *} p<0,01$; ${ }^{* *} p<0,05 ;{ }^{*} p<0,10$ 
régimen de propiedad tienen más probabilidades de realizar un desplazamiento largo al trabajo que quienes residen en viviendas de alquiler. Este resultado ya se observó en Romaní et al. (2003) e indica que los que tienen vivienda propia son más reacios a realizar un cambio en su localización residencial para minimizar el tiempo o la distancia de desplazamiento al lugar de empleo. Dada la elevada proporción de familias que, en España, son propietarias de su hogar, este resultado tiene importantes implicaciones ante cualquier medida de política laboral.

Por otro lado, y en lo que respecta al resto de variables consideradas, cabe señalar, comenzando por las características de la vivienda, que cuanto mayor es su superficie, mayor es también la probabilidad de desplazarse fuera del municipio de residencia. Este resultado encaja con el trade-off entre tamaño de la vivienda y duración del desplazamiento al trabajo descrito en el modelo seminal de Alonso (1964) y confirmado en muchos análisis empíricos posteriores (Romaní et al., 2003; Artís, Romaní y Suriñach, 2000; Romaní y Casado-Díaz, 2010; Casado-Díaz, 2000a, 2000b).

En segundo lugar, en lo que respecta a las características de los individuos en cuanto a educación y tipo de ocupación, y en concordancia con las observaciones de Simpson (1992), la cualificación profesional también incrementa las probabilidades de realizar desplazamientos largos: cada año de estudios adicional incrementa tanto la probabilidad de realizar un desplazamiento entre municipios del mismo MLT como la de realizar un desplazamiento entre diversos MLT. Por su parte, las categorías profesionales de mayor cualificación (directivos, profesionales y técnicos) son las que mayores probabilidades tienen de trabajar fuera del municipio de residencia. En cambio, los administrativos y otras categorías de cualificación similar muestran probabilidades relativamente elevadas de tener el empleo en otro municipio del mismo MLT, pero sus probabilidades de realizar un desplazamiento a otro MLT no son significativamente diferentes de las de la categoría de referencia. Dichos resultados son parecidos a los encontrados por Camarero y Oliva (2008).

Finalmente, en lo que respecta a las características del hogar y a las responsabilidades que en él pueden tener los ocupados considerados, se confirma lo concluido en otros estudios (puede consultarse un resumen de estos en Wagner y Mulder, 2015). En el caso español, Camarero y Oliva (2008) encuentran un resultado similar. Los trabajadores que, además, están al cuidado de niños o son los que mayoritariamente realizan las tareas domésticas en su hogar, significativamente tienen unas probabilidades más bajas que el resto de desplazarse fuera del municipio de residencia.

La tabla 2 recoge la estimación del modelo explicativo de la variable de cambio de residencia. En dicho modelo, la variable de interés es la otra forma de movilidad de los ocupados considerada en este artículo, la variable commuting. Tal y como puede comprobarse, los trabajadores con desplazamientos más largos al trabajo son los que más probabilidades tienen de haber realizado un cambio de residencia, un resultado que, de nuevo, es coherente con la mayoría de modelos empíricos previos (Romaní et al., 2003; Axisa et al., 2012; 
Tabla 2. Ecuación de cambio de residencia

\begin{tabular}{|c|c|c|c|c|c|c|}
\hline & \multicolumn{3}{|c|}{ Dentro del mismo MLT } & \multicolumn{3}{|c|}{ A otro MLT } \\
\hline & B & Sig. & $\operatorname{Exp}(B)$ & B & Sig. & $\operatorname{Exp}(B)$ \\
\hline Interceptación & $-1,111$ & 0,000 & & $-2,715$ & 0,000 & \\
\hline Trabaja y reside en distintos municipios del mismo MLT & 1,163 & 0,000 & 3,201 & 0,158 & 0,000 & 1,171 \\
\hline Trabaja y reside en diferentes MLT & 0,001 & 0,977 & 1,001 & 1,215 & 0,000 & 3,370 \\
\hline Trabaja y reside en el mismo municipio & \multicolumn{6}{|c|}{ Categoría de referencia } \\
\hline Edad & $-0,046$ & 0,000 & 0,955 & $-0,053$ & 0,000 & 0,948 \\
\hline Número de hijos & $-0,581$ & 0,000 & 0,559 & $-1,005$ & 0,000 & 0,366 \\
\hline Tiempo de desplazamiento al trabajo & 0,048 & 0,000 & 1,049 & 0,025 & 0,028 & 1,026 \\
\hline Años de estudios & 0,029 & 0,000 & 1,030 & 0,040 & 0,000 & 1,041 \\
\hline Hombre & 0,158 & 0,000 & 1,171 & 0,131 & 0,000 & 1,140 \\
\hline Mujer & \multicolumn{6}{|c|}{ Categoría de referencia } \\
\hline Soltero & $-0,147$ & 0,000 & 0,863 & 0,221 & 0,000 & 1,247 \\
\hline Viudo & 0,243 & 0,000 & 1,275 & 0,904 & 0,000 & 2,469 \\
\hline Separado & 0,504 & 0,000 & 1,655 & 1,142 & 0,000 & 3,134 \\
\hline Divorciado & 0,611 & 0,000 & 1,843 & 1,120 & 0,000 & 3,064 \\
\hline Casado & \multicolumn{6}{|c|}{ Categoría de referencia } \\
\hline Cuidar a un menor de 15 años: sí & 0,259 & 0,000 & 1,296 & 0,164 & 0,000 & 1,179 \\
\hline Cuidar a un menor de 15 años: no & \multicolumn{6}{|c|}{ Categoría de referencia } \\
\hline Trabajos del hogar: sí & 0,203 & 0,000 & 1,225 & 0,057 & 0,053 & 1,059 \\
\hline Trabajos del hogar: no & \multicolumn{6}{|c|}{ Categoría de referencia } \\
\hline Trabajo a tiempo completo & 0,091 & 0,000 & 1,096 & 0,055 & 0,144 & 1,057 \\
\hline Trabajo a tiempo parcial & \multicolumn{6}{|c|}{ Categoría de referencia } \\
\hline Superficie de la vivienda & 0,004 & 0,000 & 1,004 & 0,003 & 0,000 & 1,003 \\
\hline Número de habitaciones & $-0,076$ & 0,000 & 0,927 & $-0,068$ & 0,000 & 0,934 \\
\hline Número de plazas de garaje & 0,066 & 0,000 & 1,068 & 0,047 & 0,000 & 1,049 \\
\hline Número de familias en la vivienda & 0,017 & 0,853 & 1,017 & $-0,188$ & 0,180 & 0,829 \\
\hline Número de personas ocupadas en el hogar & $-0,054$ & 0,000 & 0,947 & $-0,170$ & 0,000 & 0,843 \\
\hline Número de hombres extranjeros en el hogar & $-0,302$ & 0,000 & 0,739 & 0,027 & 0,386 & 1,027 \\
\hline Número de mujeres extranjeras en el hogar & $-0,176$ & 0,000 & 0,838 & $-0,009$ & 0,775 & 0,991 \\
\hline Vivienda: propia, adquirida por compra, totalmente pagada & $-1,508$ & 0,000 & 0,221 & $-0,379$ & 0,000 & 0,685 \\
\hline Vivienda: propia, adquirida por herencia o donación & $-1,247$ & 0,000 & 0,287 & $-0,126$ & 0,037 & 0,882 \\
\hline Vivienda: alquilada & 0,060 & 0,000 & 1,062 & 1,569 & 0,000 & 4,804 \\
\hline $\begin{array}{l}\text { Vivienda: cedida gratis o a bajo precio } \\
\text { (por otro hogar, pagada por la empresa, etc.) }\end{array}$ & $-0,473$ & 0,000 & 0,623 & 0,727 & 0,000 & 2,070 \\
\hline Vivienda: otra forma de adquisición & $-0,572$ & 0,000 & 0,564 & $-0,021$ & 0,745 & 0,980 \\
\hline Vivienda: propia, por compra, con pagos pendientes (hipotecas) & \multicolumn{6}{|c|}{ Categoría de referencia } \\
\hline Calefacción central & 0,214 & 0,000 & 1,239 & 0,276 & 0,000 & 1,318 \\
\hline Calefacción individual & 0,308 & 0,000 & 1,360 & 0,292 & 0,000 & 1,339 \\
\hline Estufas & $-0,041$ & 0,031 & 0,960 & 0,204 & 0,000 & 1,226 \\
\hline Sin calefacción & \multicolumn{6}{|c|}{ Categoría de referencia } \\
\hline Tiene aseo & 0,040 & 0,597 & 1,041 & 0,170 & 0,412 & 1,185 \\
\hline No tiene aseo & \multicolumn{6}{|c|}{ Categoría de referencia } \\
\hline Baño o ducha: sí & 0,069 & 0,446 & 1,071 & $-0,328$ & 0,114 & 0,720 \\
\hline Baño o ducha: no & \multicolumn{6}{|c|}{ Categoría de referencia } \\
\hline Acceso a Internet: sí & $-0,232$ & 0,000 & 0,793 & $-0,478$ & 0,000 & 0,620 \\
\hline Acceso a Internet: no & \multicolumn{6}{|c|}{ Categoría de referencia } \\
\hline Agua corriente pública & $-0,221$ & 0,078 & 0,802 & $-0,482$ & 0,084 & 0,618 \\
\hline Agua corriente del pozo & $-0,099$ & 0,434 & 0,906 & $-0,615$ & 0,031 & 0,541 \\
\hline Sin agua corriente & \multicolumn{6}{|c|}{ Categoría de referencia } \\
\hline
\end{tabular}


Tabla 2. Ecuación de cambio de residencia (continuación)

\begin{tabular}{|c|c|c|c|c|c|c|}
\hline & \multicolumn{3}{|c|}{ Dentro del mismo MLT } & \multicolumn{3}{|c|}{ A otro MLT } \\
\hline & B & Sig. & $\operatorname{Exp}(B)$ & B & Sig. & $\operatorname{Exp}(B)$ \\
\hline Estado del edificio: ruinoso & $-0,192$ & 0,077 & 0,826 & $-0,924$ & 0,040 & 0,397 \\
\hline Estado del edificio: malo & $-0,109$ & 0,090 & 0,897 & $-0,439$ & 0,025 & 0,645 \\
\hline Estado del edificio: deficiente & $-0,201$ & 0,000 & 0,818 & 0,086 & 0,160 & 1,090 \\
\hline Estado del edificio: bueno & \multicolumn{6}{|c|}{ Categoría de referencia } \\
\hline Con ascensor & $-0,480$ & 0,000 & 0,619 & $-0,247$ & 0,000 & 0,781 \\
\hline Sin ascensor & \multicolumn{6}{|c|}{ Categoría de referencia } \\
\hline Con accesibilidad & 0,113 & 0,000 & 1,120 & 0,117 & 0,000 & 1,124 \\
\hline Sin accesibilidad & \multicolumn{6}{|c|}{ Categoría de referencia } \\
\hline Con gas & $-0,025$ & 0,011 & 0,975 & $-0,258$ & 0,000 & 0,772 \\
\hline Sin gas & \multicolumn{6}{|c|}{ Categoría de referencia } \\
\hline Agua caliente central: sí & $-0,049$ & 0,000 & 0,952 & 0,003 & 0,911 & 1,003 \\
\hline Agua caliente central: no & \multicolumn{6}{|c|}{ Categoría de referencia } \\
\hline Administrativos & 0,002 & 0,914 & 1,002 & $-0,203$ & 0,000 & 0,816 \\
\hline $\begin{array}{l}\text { Artesanos y trabajos cualificados de las industrias } \\
\text { manufactureras y de la construcción }\end{array}$ & $-0,068$ & 0,000 & 0,935 & $-0,203$ & 0,000 & 0,816 \\
\hline Directivos & 0,050 & 0,026 & 1,052 & 0,068 & 0,362 & 1,070 \\
\hline Ocupaciones elementales & $-0,131$ & 0,000 & 0,877 & $-0,171$ & 0,002 & 0,843 \\
\hline Operadores de instalaciones y maquinaria, y montadores & $-0,088$ & 0,000 & 0,916 & $-0,169$ & 0,011 & 0,844 \\
\hline Técnicos y profesionales científicos e intelectuales & $-0,031$ & 0,085 & 0,969 & 0,073 & 0,169 & 1,076 \\
\hline Técnicos; profesionales de apoyo & 0,037 & 0,025 & 1,038 & $-0,043$ & 0,402 & 0,958 \\
\hline $\begin{array}{l}\text { Trabajadores cualificados en el sector agrícola, ganadero, } \\
\text { forestal y pesquero }\end{array}$ & $-0,126$ & 0,002 & 0,882 & $-0,231$ & 0,026 & 0,794 \\
\hline $\begin{array}{l}\text { Trabajador de los servicios de restauración, personales, } \\
\text { protección y vendedor }\end{array}$ & Categoría & de refer & ncia & & & \\
\hline
\end{tabular}

Fuente: Censo de Población 2011, INE

Nota: Nivel de significación: ${ }^{\star \star \star} p<0,01 ;{ }^{* \star} p<0,05 ;{ }^{*} p<0,10$

Newbold y Scott, 2013; Mendiola et al., 2014; Brown et al., 2015). Por otro lado, los trabajadores que han cambiado de residencia recientemente tienden a vivir en domicilios más grandes y mejor equipados que los que siguen en la misma. Este resultado parece indicar que los motivos de las mudanzas están más relacionados con el intento de mejorar la calidad de vida residencial que con disminuir el tiempo o la distancia al trabajo, lo cual es coherente con la hipótesis del intervalo de indiferencia en el tiempo de desplazamiento propuesta por Vale (2013). En cambio, el régimen de propiedad del domicilio no parece una variable significativa en la ecuación del cambio de residencia, al contrario de lo que ocurría en la ecuación de commuting.

Cabe destacar también que, de manera similar a lo que ocurría en la ecuación de commuting, los trabajadores más educados y cualificados son los que más probabilidades tienen de cambiar su lugar de residencia (un resultado coherente con el obtenido en el trabajo de Arévalo et al., 2008). Por su parte, los empleados de cualificación media, como los administrativos, tienen probabilidades relativamente elevadas de cambiar de municipio de 
residencia dentro del mismo MLT, pero bajas de ir a residir a otro MLT. Un comportamiento similar presentan los trabajadores inmigrantes. Finalmente, el número de miembros del hogar parece incentivar los cambios de residencia: cuanto más grande es el tamaño de la unidad familiar, más probabilidades hay de que se produzca una variación en el municipio o en el MLT de residencia.

En resumen, debe destacarse que los resultados muestran claramente la importancia de considerar el peso que cada una de las formas de movilidad (desplazamiento al trabajo y migración y/o cambio residencial) tiene a la hora de explicar a la otra, y que, por tanto, dicha influencia mutua debe reconocerse en el momento de formular modelos que permitan aumentar el conocimiento sobre los factores que las determinan. Este resultado apunta, por tanto, a la necesidad de operar con cautela al proponer y discutir modelos explicativos cuando no se dispone de información apropiada y referida a las áreas geográficas relevantes.

\section{Conclusiones}

Este estudio analiza dos formas de movilidad de los ocupados: el desplazamiento al trabajo, commuting, y la migración o movilidad residencial. Lo hace partiendo de la hipótesis de que ambos tipos de movilidad se influyen mutuamente y de que existe un conjunto de variables explicativas comunes que muestran efectos distintos en uno y otro caso.

Se trata de dos formas de movilidad que influyen de manera significativa en el bienestar individual y social (en aspectos tales como la forma urbana, los transportes o el medio ambiente) y que, en consecuencia, han dado lugar a una abundante literatura, tanto por separado como en cuanto a la relación que se establece entre ellas. Se trata de aportaciones que, sin embargo, no han generado ningún consenso, debido a que, a menudo, los resultados alcanzados difieren de unos territorios a otros y se dan en función de los marcos teóricos, las variables y los métodos estadísticos utilizados, de tal forma que los resultados alcanzados distan de ser concluyentes. La necesidad de abordar estos fenómenos se acentúa, además, en el caso español, para el que no se dispone de demasiadas evidencias y, en cualquier caso, son necesarios análisis actualizados y adaptados a las nuevas circunstancias laborales y sociales. Cabe destacar que el análisis realizado incorpora una aportación en términos metodológicos, al prestar especial atención a la cuestión de la unidad territorial de referencia utilizada. Son escasas las investigaciones previas en este campo basadas en geografías de tipo funcional, no administrativo, que permitan, por ejemplo, distinguir entre movilidad residencial y migraciones. En nuestro caso, se usa una nueva base de microdatos específicamente recodificada por el INE y apropiada para este estudio, ya que se utilizan mercados locales de trabajo españoles como unidad territorial.

Los resultados, obtenidos a partir del análisis de una muestra de microdatos procedentes del Censo de Población de 2011, muestran una relación 
clara entre el commuting y el cambio de residencia de los trabajadores y de las familias en España. Dicha relación, similar a la encontrada en otros estudios que exploran el caso español o el de otros países, indica que los cambios de residencia tienden a alejar a los trabajadores de su lugar de empleo, en vez de acortar los desplazamientos hacia este. Las razones de los cambios del lugar de residencia parecen relacionadas, predominantemente, con la posibilidad de adquirir una vivienda más grande, de construcción más reciente y mejor equipada.

Entre los resultados alcanzados, cabe destacar algunos. En primer lugar, los trabajadores más cualificados aparecen como los más móviles, no solo en lo que se refiere al commuting, sino también a los cambios de residencia. Por su parte, las características familiares parecen desempeñar un papel fundamental en la explicación de la movilidad, tanto si se refiere a la cotidiana como a la de carácter residencial. Así, los trabajadores con mayores responsabilidades familiares (cuidado de niños o tareas del hogar) son los que menos probabilidades tienen de realizar desplazamientos largos en su trayecto del hogar al lugar de empleo, mientras que las familias más numerosas son las que menor probabilidad tienen de cambiar su municipio de residencia. Finalmente, el régimen de propiedad de la vivienda también influye en la decisión de los trabajadores de realizar desplazamientos laborales o no.

En suma, el análisis llevado a cabo permite contrastar, por una parte, la permanencia de factores que ya habían sido apuntados como relevantes en la literatura sobre movilidad de los ocupados, tanto en el ámbito internacional como en el español, pero, por otro lado, también nos revelan la existencia de una evolución en la forma en que dichos factores influyen sobre las variables de interés. Dicha evolución está asociada, con toda probabilidad, a los procesos de cambio que tienen lugar en las pautas de comportamiento en referencia a la ubicación de los lugares de residencia y de trabajo que se vienen observando en las últimas décadas, además de los cambios acaecidos en las infraestructuras de transporte y las transformaciones experimentadas por los hogares españoles. Asimismo, estos cambios pueden estar muy influidos por el momento del ciclo económico en el que fueron recogidos los datos utilizados en este análisis, y que se corresponden con una de las peores crisis financieras y sociales que ha sufrido la sociedad contemporánea española, lo que invita a ulteriores investigaciones que revisen las relaciones observadas en el artículo conforme vayan estando disponibles nuevas fuentes de datos que permitan estudiar lo acaecido en los años posteriores.

\section{Referencias bibliográficas}

Aguilera, A. y Mignot, D. (2004). «Urban sprawl, polycentrism and commuting: A comparison of seven French urban areas». Urban Public Economics Review, 1, 93-113.

Alonso, W. (1964). Location and Land Use: Towards a General Theory of Land Rent [en línea]. Cambridge, MA: Harvard University Press.

<https://doi.org/10.4159/harvard.9780674730854>. 
Arévalo, R.; Ferrero, L.; Otero, M.S.; Uña, J. de (2008). Movilidad residencial en España: Un análisis longitudinal [en línea]. Documentos de trabajo del Departamento de Economía Aplicada de la Universidad de Vigo. <http://webs.uvigo. $\mathrm{es} / \mathrm{x} 06 />$.

Artís, M.; Romaní, J. y SuriñaCH, J. (2000). «Determinants of Individual Commuting in Catalonia, 1986-91: Theory and Empirical Evidence». Urban Studies, 37 (8), 1431-1450.

$<$ https://doi.org/10.1080/00420980020080191>.

AXISA, J.J.; NewbOLD, K.B. y SCOTT, D.M. (2012). «Migration, urban growth and commuting distance in Toronto's commuter shed». Area [en línea], 44 (3), 344355 . <https://doi.org/10.1111/j.1475-4762.2012.01097.x>.

BLOZE, G. y SKAK, M. (2016). «Housing equity, residential mobility and commuting». Journal of Urban Economics [en línea]. <https://doi.org/10.1016/j.jue.2016.09.003>.

BorJas, G.; Bronars, S. y TREJO, S. (1992). «Self-selection and internal migration in the United States». Journal of Urban Economics [en línea], 32, 159-185. <https://doi.org/10.1016/0094-1190(92)90003-4>.

Brown, D.L.; Champion, T.; Coombes, M. y Wymer, C. (2015). "The Migration-commuting nexus in rural England: A longitudinal analysis». Journal of Rural Studies [en línea], 41, 118-128. <https://doi.org/10.1016/j.jrurstud.2015.06.005>.

Camarero, L.A. y Oliva, J. (2008). «Exploring the social face of urban mobility: Daily mobility as part of the social structure in Spain». International Journal of Urban and Regional Research [en línea], 32 (2), 344-362. <https://doi.org/10.1111/j.1468-2427.2008.00778.x>.

CASADO-DíAZ, J.M. (2000a). Diferencias de género en los desplazamientos cotidianos por razones laborales. Documento de trabajo del IVIE, WP-EC 2000-03, Valencia.

- (2000b). "Local Labour Market Areas in Spain: A Case Study». Regional Studies [en línea], 34 (9), 843-856. <https://doi.org/10.1080/00343400020002976>.

- (2001). "Who are the commuters?: A microdata analysis of travel-to-work». En: Columbus, F. (ed.). European Economic and Political Issues. Nueva York: Nova Science Publishers, 25-50.

Casado, J.M.; Martínez, L. y Flórez, F. (2010): «Los mercados locales de trabajo españoles: Una aplicación del nuevo procedimiento británico». En: AlBerTos, J.M. y FERIA, J.M. (ed.). La ciudad metropolitana en España: Procesos urbanos en los inicios del siglo XXI. Madrid. Thomson-Civitas, 275-313.

Casado-Díaz, J.M. y CoOmbes, M. (2011). «The delineation of 21st Century local labour market areas: A critical review and a research agenda». Boletín de la Asociación de Geógrafos Españoles, 57, 7-32.

Casado-Díaz, J.M.; Martínez-Bernabéu, L. y Flórez-Revuelta, F. (2016). «Automatic parameter tuning for functional regionalisation methods». Papers in Regional Science [en línea]. <https://doi.org/10.1111/pirs.12199>.

Casado-Díaz, J.M.; Martínez-Bernabeu, L. y Rowe, F. (2017). «An Evolutionary Approach to the Delimitation of Labour Market Areas: An Empirical Application for Chile». Spatial Economic Analysis [en línea]. <https://doi.org/10.1080/17421772.2017.1273541>. 
Coombes, M.G. y Bond, S. (2008). Travel-to-Work Areas: The 2007 Review. Londres. Office for National Statistics.

Coombes, M.G.; Casado-Díaz, J.M.; Martínez-Bernabeu, L. y Carausu, F. (2012). Study on comparable Labour Market Areas [en línea]. Final Research Report, Eurostat Specific Contract 50405.2010.004-2011.325. <https://ec.europa. eu/eurostat/cros/content/study-comparable-labour-market-areas_en>.

Coombes, M.G.; Green, A.E. y Openshaw, S. (1986). "An efficient algorithm to generate official statistical reporting areas: The case of the 1984 Travel-toWork Areas revision in Britain». Journal of the Operational Research Society, 37, 943-953. <https://doi.org/10.1057/jors.1986.163>.

FAGGIAN, A. y MCCANN, P. (2009). «Universities, agglomerations and graduate human capital mobility». Tijdschrift voor Economische en Sociale Geografie [en línea], 100 (2), 210-223. <https://doi.org/10.1111/j.1467-9663.2009.00530.x>.

Feria-Toribio, J.M.; Casado-Díaz, J.M. y Martínez-Bernabeu, L. (2015). «Inside the metropolis: The articulation of Spanish metropolitan areas into local labor markets». Urban Geography [en línea], 36 (7), 1018-1041. $<$ https://doi.org/10.1080/02723638.2015.1053199>.

GoOdman, J.F.B. (1970). "The definition and analysis of local labour markets: Some empirical problems». British Journal of Industrial Relations [en línea], 8, 179-186. <https://doi.org/10.1111/j.1467-8543.1970.tb00968.x>.

Greene, W.H. (2012). Econometric Analysis. 7. a ed. Nueva York. Pearson.

GREENWOOD, M. (1969). «An analysis of the determinants of geographic labor mobility in the United States». Review of Economics and Statistics [en línea], 51, 189-194. $<$ https://doi.org/10.2307/1926728>.

Greenwood, M. y HunT, G. (1989). «Jobs versus amenities in the analysis of metropolitan migration». Journal of Urban Economics [en línea], 25, 1-16. <https://doi.org/10.1016/0094-1190(89)90040-5>.

- (2003). «The Early History of Migration Research». International Regional Science Review [en línea], 26 (1), 3-37. <https://doi.org/10.1177/0160017602238983>.

Gujarati, D. y PORTER, D. (2010). Econometría. 5.a ed. Barcelona. McGraw-Hill Interamericana.

Han, Y.; Goetz, S.J.; KIm, T. y LeE, J. (2013). «Estimating Employment-Related Migration from Overlapping Migration and Commuting Networks». Growth and Change [en línea], 44 (3), 474-493. $<$ https://doi.org/10.1111/grow.12016>.

KnAPP, T. y Graves, P. (1989). "On the role of amenities in models of migration and regional development». Journal of Regional Science [en línea], 29, 71-87. <https://doi.org/10.1111/j.1467-9787.1989.tb01223.x>.

Marmolejo, C. y Tornés, M. (2015). «¿Reduce el policentrismo la movilidad laboral?: Un análisis para las siete grandes áreas metropolitanas en España». Scripta Nova, 18 (500), 1-30.

MARTíneZ-BERnABÉU, L. y CASADO-DíAZ, J.M. (2016). «Delineating zones to increase geographical detail in individual response data files: An application to the Spanish 2011 Census of population». Moravian Geographical Reports [en línea], 24 (2), 26-36.

<https://doi.org/10.1515/mgr-2016-0008>. 
Martínez-Bernabeu, L.; Flórez-Revuelta, F. y Casado-Díaz, J.M. (2012). "Grouping genetic operators for the delineation of functional areas based on spatial interaction». Expert Systems with Applications [en línea], 39 (8), 6754-6766. $<$ https://doi.org/10.1016/j.eswa.2011.12.026>.

Mendiola, L.; González, P. y Cebollada, A. (2014). "The link between urban development and the modal split in commuting: The case of Biscay». Journal of Transport Geography [en línea], 37, 1-9. $<$ https://doi.org/10.1016/j.jtrangeo.2014.03.014>.

Molho, I. (1986). "Theories of migration: A review». Scottish Journal of Political Economy [en línea], 33 (4), 396-419. <https://doi.org/10.1111/j.1467-9485.1986.tb00901.x>.

Mueser, P. y GraVES, P. (1995). «Examining the role of economic opportunity and amenities in explaining population distribution». Journal of Urban Economics [en línea], 37, 176-200. $<$ https://doi.org/10.1006/juec.1995.1010>.

Newbold, K.B. y SCOTT, D.M. (2013). «Migration, commuting distance, and urban sustainability in Ontario's Greater Golden Horseshoe: Implications of the Greenbelt and Places to Grow legislation». The Canadian Geographer / Le Géographe Canadien [en línea], 57 (4), 474-487. $<$ https://doi.org/10.1111/j.1541-0064.2013.12044.x>.

PINO, J.A. del (2014). «Sociology of residence and mobile residence: Theoretical advances and practical limits / Sociología de la residencia y residencia móvil: Logros teóricos y limítes prácticos». Empiria [en línea], 27, 21-48. <http://dx.doi.org/10.5944/empiria.27.2014.10861>.

- (2015). Estructuras residenciales y movilidad: Más allá de la segunda residencia. Madrid: CIS.

Romaní, J. y CASADO-DíAZ, J.M. (2010). «Movilidad por razón de trabajo en áreas metropolitanas: Un análisis basado en datos individuales». En: AlBERTOS, Juan Miguel y Feria, José María (eds.). La ciudad real en España: Procesos urbanos y metropolitanos en la primera década del siglo XXI. Madrid: Thomson-Civitas.

Romaní, J.; Suriñach, J. y ArTís, M. (2003). "Are Commuting and Residential Mobility Decisions Simultaneous?: The Case of Catalonia, Spain». Regional Studies [en línea], 37 (8), 813-826. <https://doi.org/10.1080/0034340032000128730>.

SHUAI, X. (2012). «Does Commuting Lead to Migration?». Journal of Regional Analysis and Police (JRAP), 42 (3), 237-250.

SimPSON, Wayne (1992). Urban structure and the labour market. Oxford. Clarendon Press.

VALE, D.S. (2013). «Does commuting time tolerance impede sustainable urban mobility?: Analysing the impacts on commuting behaviour as a result of workplace relocation to a mixed-use centre in Lisbon». Journal of Transport Geography [en línea], 32, 38-48. <https://doi.org/10.1016/j.jtrangeo.2013.08.003>.

Wagner, M. y Mulder, C.H. (2015). «Spatial Mobility, Family Dynamics, and Housing Transitions». Köln Z Soziol (supl.) [en línea], 67, 111-135. <https://doi.org/10.1007/978-3-658-11490-9_6>.

Wrede, M. (2013). «Heterogeneous skills, migration, and commuting». Papers in Regional Science [en línea], 92 (2).

<https://doi.org/10.1111/j.1435-5957.2012.00471.x>. 
ZAX, J. (1991). "Compensation for commutes in labour and housing markets». J. Urban Econ. [en línea], 30, 192-207. <https://doi.org/10.1016/0094-1190(91)90036-7>.

- (1994). «When is a move a migration?». Reg. Sci. \& Urban Econ. [en línea], 24, 341-360. <https://doi.org/10.1016/0166-0462(93)02034-Z>.

ZaX, J. y KaIN, J. (1991). "Commutes, quits and moves». J. Urban Econ. [en línea], 29, 153-165.

<https://doi.org/10.1016/0094-1190(91)90010-5>. 